\title{
Analysis of data for stored polarized beams using a spin flipper
}

\author{
Yu. M. Shatunov \\ Budker Institute of Nuclear Physics, Novosibirsk, 630090, Russia \\ S. R. Mane* \\ Convergent Computing, Inc., P.O. Box 561, Shoreham, New York 11786, USA
}

(Received 22 April 2008; published 24 September 2008)

\begin{abstract}
We employ the so-called "spin response formalism," which is a linear response theory applied to the spin dynamics in circular accelerators, to analyze recent measurements of spin-flip resonance widths. The data was taken using a radial field rf dipole spin flipper to flip the spins of stored polarized proton and deuteron beams at the COSY storage ring. Numerical calculations are presented, which provide a satisfactory fit to the data.
\end{abstract}

DOI: 10.1103/PhysRevSTAB.11.094002

PACS numbers: 29.27.Hj, 29.20.D-, 02.60.Lj

\section{INTRODUCTION}

Several papers have been recently published describing spin-flip studies for stored polarized beams, using both protons [1-3] and deuterons [4,5]. A radial field rf dipole spin flipper was used to flip the spins. The authors (the SPIN@COSY Collaboration) have claimed to find "unexpected discrepancies" between the measured values and the theoretical expressions for the spin-flip resonance widths [3,5]. Some errors in the theoretical analysis by the SPIN@COSY Collaboration were pointed out by Bai, MacKay, and Roser [6] (which fortunately have now been acknowledged by the collaboration). Nevertheless, other discrepancies remain, as advertised in a recent paper [5]. Specifically, the theoretical expressions for the resonance strength in [1-5] did not account for the full spin-flip contributions of a radial field rf dipole spin flipper. In particular, in addition to its direct spin coupling, an rf dipole spin flipper also excites coherent vertical betatron oscillations, and these can in turn couple to the spin, thereby modifying the overall spin-flip resonance strength. This latter contribution was omitted in [1-5].

In this paper, we employ the so-called "spin response formalism," which is a linear response theory applied to the spin dynamics in circular accelerators [7-9], to analyze the data in some of the above papers, specifically [3] (protons) and [5] (deuterons). The spin response functions are calculated by the computer code ASPIRRIN (Analysis of SPIn Resonances in RINgs) [8,9]. We used ASPIRRIN to fit the proton and deuteron spin-flip data in [3,5], respectively. A paper to fit the COSY spin-flip studies, for stored polarized deuterons and an rf dipole, has also been written by Kondratenko, Kondratenko, and Filatov [10] (in Russian), but the above paper does not treat the polarized proton data.

*srmane@optonline.net
The structure of this paper is as follows. In Sec. II, we present the basic theoretical formalism. In Sec. III, we present our numerical calculations for the COSY spinflip data. Section IV concludes.

\section{BASIC FORMALISM}

We treat a particle of charge $e$, mass $m$, with velocity $\boldsymbol{v}$ and spin $s$. The Lorentz factor is $\gamma=1 / \sqrt{1-v^{2} / c^{2}}$. The magnetic moment anomaly will be denoted by $G=(g-$ 2) $/ 2$. In particular, $G_{p} \simeq 1.792847$ for protons and $G_{d} \simeq$ -0.142987 for deuterons. The externally prescribed electric and magnetic fields of the accelerator will be denoted by $\boldsymbol{E}$ and $\boldsymbol{B}$, respectively. We employ cgs units. For motion along the reference (or design) orbit of the storage ring (i.e. along the guide field of the accelerator), we denote the magnetic rigidity by $B \rho=p_{0} c / e$, where $p_{0}$ is the reference momentum. We shall use the arclength $s$ along the reference orbit as the independent variable, and define the azimuth $\theta=s / R$, where the ring circumference is $2 \pi R$. We employ a prime to denote differentiation with respect to $\theta$, so $f^{\prime} \equiv d f / d \theta$ for any function $f$. Our coordinate system is $(\hat{\boldsymbol{x}}, \hat{\boldsymbol{y}}, \hat{z})$, a right-handed orthogonal system where $\hat{\boldsymbol{x}}$ is radially outward, $\hat{\boldsymbol{y}}$ is along the reference orbit, and $\hat{z}=\hat{\boldsymbol{x}} \times \hat{\boldsymbol{y}}$ (this is vertical in a planar ring). The positive sense of circulation is counterclockwise around the ring. We denote the horizontal and vertical coordinates of a particle by $x$ and $z$, respectively. We scale the orbital variables to be dimensionless, so what we call " $x$ " would generally be denoted by $x / R$ by other authors. The horizontal and vertical betatron tunes will be denoted by $\nu_{x}$ and $\nu_{z}$, respectively. We also define the dimensionless scaled magnetic fields on the reference orbit $K_{x, y, z}=B_{x, y, z} / B_{0}$, where $B_{0}$ is a reference value given by the arc dipoles

$$
B_{0}=\frac{1}{2 \pi} \int_{\operatorname{arcs}} B_{z} d \theta
$$

We introduce the important phase $\Theta$, defined via 


$$
\Theta=\int_{0}^{\theta} K_{z}(\tilde{\theta}) d \tilde{\theta}=\int_{0}^{\theta} \frac{R}{\rho(\tilde{\theta})} d \tilde{\theta},
$$

where $\rho$ is the local bend radius in the horizontal plane. The spin precession equation of motion in the externally prescribed electric and magnetic fields of the accelerator is known as the Thomas-BMT equation $[11,12]$. Using $\theta$ as the independent variable, the equation takes the form

$$
\frac{d s}{d \theta}=\boldsymbol{W} \times \boldsymbol{s} .
$$

We subdivide the spin precession vector $\boldsymbol{W}$ into two parts $\boldsymbol{W}=\boldsymbol{W}_{0}+\boldsymbol{w}$, where $\boldsymbol{W}_{0}$ denotes the precession vector on the reference orbit, and $\boldsymbol{w}$ denotes all of the other terms. We treat $\boldsymbol{w}$ as a perturbation. We define the symbol $\nu_{0}=G \gamma_{0}$. Here $\gamma_{0}$ (a constant) is the value of $\gamma$ on the reference orbit. For a planar ring such as COSY, $\boldsymbol{W}_{0}=\nu_{0} K_{z} \hat{z}$ is vertical. The detailed expression for $\boldsymbol{w}$ is not required here; it can be found, for example, in the review article [13]. On the reference orbit, the spins precess around the vertical axis with a precession tune called the "spin tune" $\nu$. We distinguish between $\nu$ and $\nu_{0}$ for the sake of the FroissartStora formula (see the next paragraph).

Froissart and Stora [14] solved the spin precession equation in a planar ring, for the passage across a single isolated resonance. Suppose the beam is initially vertically polarized, and the beam energy is increased at a uniform rate so that $\nu=G \gamma=\nu_{\text {res }}+\alpha \theta$, where $\nu_{\text {res }}$ is the resonant spin tune, $\alpha$ is a constant, and the spin tune is increased from far below to far above the resonance. Froissart and Stora calculated the final asymptotic vertical polarization, and obtained the result

$$
\frac{P_{f}}{P_{i}}=2 e^{-\pi|\varepsilon|^{2} /(2|\alpha|)}-1
$$

Here $P_{i}$ and $P_{f}$ are the initial and final vertical polarizations, respectively. Equation (2.4) is called the FroissartStora formula. The value of $|\varepsilon|$ is called the resonance strength. It is given by

$$
\begin{aligned}
\varepsilon= & \left\langle\left[(G \gamma+1) z^{\prime \prime}+i(G \gamma-G) K_{z} z^{\prime}\right.\right. \\
& \left.\left.-i(1+G) K_{z}^{\prime} z\right] e^{i G \gamma \Theta}\right\rangle
\end{aligned}
$$

The above integral coincides (up to differences of notation) with that given in [15]. Here $G \gamma$ is evaluated at the center of the resonance, i.e. $G \gamma=\nu_{\text {res }}$, and we shall take this as the value of $\nu_{0}$ below. The angle brackets denote the average

$$
\langle f(\theta)\rangle \equiv \lim _{T \rightarrow \infty}\left\{\frac{1}{2 T} \int_{-T}^{T} f(\theta) d \theta\right\} .
$$

The average in Eq. (2.6) picks out the nonoscillating component of $f(\theta)$, if it exists (else the average is zero). We can use the average in Eq. (2.6) to establish the following result:

$$
0=\left\langle\left(z^{\prime} e^{i G \gamma \Theta}\right)^{\prime}\right\rangle=\left\langle\left(z^{\prime \prime}+i G \gamma K_{z} z^{\prime}\right) e^{i G \gamma \Theta}\right\rangle .
$$

The proof is self-evident because the integrand is a perfect derivative of a bounded function.

The Froissart-Stora formula [Eq. (2.4)] is also applicable for spin flippers, where now the beam energy is held fixed and instead the frequency (or tune) of the spin flipper is swept across the spin tune. The value of $z$ in Eq. (2.5) is given by the coherent betatron oscillations induced by the rf dipole spin flipper. The term in $K_{z}^{\prime}$ in Eq. (2.5) is a fringe field term from the edges of the horizontal dipoles. We can use Eq. (2.7) to eliminate $z^{\prime \prime}$ :

$$
\varepsilon=-i\left\langle\left[\left(\nu_{0}^{2}+G\right) K_{z} z^{\prime}+(1+G) K_{z}^{\prime} z\right] e^{i G \gamma \Theta}\right\rangle .
$$

Note that the integral in Eq. (2.5) contains a term $z^{\prime \prime}$ which is nonzero in any element where the orbit has curvature, e.g., quadrupoles, whereas the integral in Eq. (2.8) receives contributions only from elements where the bending field is nonzero on the design orbit (i.e. the horizontal dipoles and their fringe fields). It is therefore impossible to say which set of beam line elements (dipoles or quadrupoles) dominate the contribution to the resonance strength.

We performed our numerical calculations using the program ASPIRRIN [8,9], which makes use of the spin response formalism [7-9]. Let the rf dipole spin flipper be located at $\theta_{0}$ (mathematically, a $\delta$ function), and let its integrated field be

$$
\int B_{\mathrm{rf}} d \ell=B_{\mathrm{osc}} L \cos \left(\nu_{\mathrm{rf}} \theta+\chi\right) .
$$

Here $\nu_{\text {rf }}$ is the tune of the spin flipper, $\chi$ is an initial phase, and $B_{\mathrm{osc}} L$ denotes the peak integrated field. The spin response formalism gives the resonance strength as $[7,8]$

$$
\varepsilon=\frac{1}{4 \pi} \frac{B_{\mathrm{osc}} L}{B \rho}\left|F_{3}\left(\theta_{0}\right)\right|
$$

where for a planar ring such as COSY,

$$
\begin{aligned}
F_{3}\left(\theta_{0}\right)= & \frac{1}{2}\left\{\frac { f _ { z } ( \theta _ { 0 } ) } { e ^ { i 2 \pi ( \nu - \nu _ { z } ) } - 1 } \int _ { \theta _ { 0 } } ^ { \theta _ { 0 } + 2 \pi } \left[\left(\nu_{0}^{2}+G\right) K_{z} f_{z}^{* \prime}\right.\right. \\
& \left.+(1+G) K_{z}^{\prime} f_{z}^{*}\right] e^{i \nu_{0} \Theta} d \theta \\
& -\frac{f_{z}^{*}\left(\theta_{0}\right)}{e^{i 2 \pi\left(\nu+\nu_{z}\right)}-1} \int_{\theta_{0}}^{\theta_{0}+2 \pi}\left[\left(\nu_{0}^{2}+G\right) K_{z} f_{z}^{\prime}\right. \\
& \left.\left.+(1+G) K_{z}^{\prime} f_{z}\right] e^{i \nu_{0} \Theta} d \theta\right\} .
\end{aligned}
$$

Here $f_{z}=\sqrt{\beta_{z} / R} e^{i \phi_{z}}$, where $\beta_{z}$ is the vertical beta function and the phase is given by $d \phi_{z} / d \theta=R / \beta_{z}$. Also recall $\nu_{0}=G \gamma_{0}$ and we evaluate the integral at $\nu_{0}=\nu_{\text {res }}$. The above expression (in the approximation of ultrarelativistic electrons) was first derived in [16]. The spin response formalism [8,9] actually derives a set of functions $F_{1}-F_{5}$, and for general rings. The function of relevance to us is $F_{3}$, and for a planar uncoupled ring its value is equal to the above expression. The value of $\chi$ does not contribute to the 
resonance strength for a ring with only one spin flipper, but would be relevant if there were multiple spin flippers.

\section{NUMERICAL CALCULATIONS FOR COSY}

We shall now apply the spin response formalism to analyze the spin-flip resonance strength for a radial field rf dipole for a model of the COSY storage ring. We performed our numerical computations using a datafile of the COSY machine optics, and the ASPIRRIN program [8,9], with extensions written by one of the authors (Mane). Spinflip studies for polarized protons and deuterons at COSY, using an rf dipole, were reported in [1-5]. For polarized protons, the data in $[1,2]$ mainly treat spin-flip efficiency and report only one resonance width measurement in each paper. We shall fit the polarized proton studies in [3], because this paper reports numerous measurements of resonance strengths (specifically, the resonance strength is plotted as a function of the vertical betatron tune). The stored polarized proton beam momentum was $p=$ $2.1 \mathrm{GeV} / c$. The polarized deuteron data was taken at a momentum $p=1.85 \mathrm{GeV} / c[4,5]$. We shall fit the data in [5] because it is more detailed (Ref. [5] also displays the resonance strength plotted as a function of the vertical betatron tune).

There are some caveats to note first, however, before we display our theoretical computations. The studies reported in [1-5] were performed using a variety of machine optics. We do not have detailed information for every study. COSY is a racetrack storage ring with two $180^{\circ}$ arcs and two long straight sections. (The COSY footprint, including the rf dipole and external beam lines, is displayed, for example, in Fig. 4 of [3].) For brevity we shall henceforth write "COSY" instead of "our model of COSY," but the above fact should be understood in all of our calculations. During machine operations, the vertical betatron tune in COSY is varied by adjusting the arc quadrupoles. In the graphs below plotting the value of $\left|F_{3}\right|$ as a function of the vertical betatron tune, we recomputed $F_{3}$ (recomputed the optics) for each setting of the quadrupoles, but again we do not have precise information about the actual COSY machine operations.

The next caveat to note is that we treat the beam as monoenergetic. It was reported in [3] (for protons) and in [5] (for deuterons) that the respective spin-flip resonance strengths did not depend on the beam momentum spread or the vertical beam size. Note also that the rf cavities were shorted at flattop during the spin-flip studies, so there were no synchrotron oscillations.

Next, in both [3,5], the quantity displayed is the ratio

$$
\mathcal{R}=\frac{\varepsilon_{F S}}{\varepsilon_{0}},
$$

where $\varepsilon_{F S}$ is the experimentally measured spin-flip resonance width and the "direct" coupling of an rf dipole to the spin is

$$
\varepsilon_{0}=\frac{G \gamma+1}{4 \pi} \frac{B_{\mathrm{osc}} L}{B \rho} .
$$

Hence the value of $\left|F_{3}\right|$ must be compared to the quantity

$$
\mathcal{F}=(G \gamma+1) \mathcal{R}
$$

Actually, there is a further complication because the SPIN@COSY collaboration made factor of 2 errors in their analysis; this was corrected by Bai, MacKay, and Roser [6], and we shall discuss factors of 2 below.

We stated in Eq. (2.10) that the resonance strength is proportional to $\left|F_{3}\left(\theta_{\mathrm{rf}}\right)\right|$, i.e., the resonance strength depends on the location of the $\mathrm{rf}$ dipole around the ring circumference. The value of $F_{3}$ depends not only on the particle species, but also on the beam momentum and the ring optics (hence the vertical betatron tune). We plot the value of $\left|F_{3}\right|$ around the COSY ring in Fig. 1 for protons (left) and deuterons (right). For the protons, we used $p=$ $2.1 \mathrm{GeV} / c$ and $\nu_{z}=3.525$ [3], and for deuterons $p=$ $1.85 \mathrm{GeV} / c$ and $\nu_{z}=3.60$ [5]. Note that $\left|F_{3}\right| \gg 1$ for protons but $\left|F_{3}\right|<1$ everywhere for deuterons. In particular, for deuterons the rf dipole seems to be located at a point where $\left|F_{3}\right|$ attains almost its minimum value.

The large value of $\left|F_{3}\right|$ for protons can be understood because $G \gamma \simeq 4.395$ for $p=2.1 \mathrm{GeV} / c$, and the vertical betatron tune is $\nu_{z}=3.525$, so $8-\nu_{z} \simeq 4.475$, so $\mid \nu_{0}-$ $\left(8-\nu_{z}\right) \mid \simeq 0.08$. Hence for protons, $F_{3}$ contains a small resonance denominator. For deuterons, $G \gamma \simeq-0.20084$ for $p=1.85 \mathrm{GeV} / c$, and $\nu_{z}=3.60$, so $\nu_{z}-4 \simeq-0.4$, so $\left|\nu_{0}-\left(\nu_{z}-4\right)\right| \simeq 0$. 2 . Hence, for deuterons, $F_{3}$ does not contain such a small resonance denominator. Also, the values of the coefficients in Eq. (2.11) are $\nu_{0}^{2}+G \simeq 21.1$ and $1+G \simeq 2.792847$ for protons, but only $\nu_{0}^{2}+G \simeq$ -0.1 and $1+G \simeq 0.857013$ for deuterons.

Note also that for deuterons with $G \gamma \simeq-0.2$, the contribution to $F_{3}$ is dominated by the fringe field term $K_{z}^{\prime}$ in Eq. (2.11). We have employed a simple "hard edge" model for the fringe field, and it is possible that a more sophisticated model of the fringe field would yield a better fit to the data. For protons, however (with $G \gamma \simeq 4.395$ ), the contribution to $F_{3}$ is dominated by the term in $K_{z}$ in Eq. (2.11). This comes from the body of a dipole and is unambiguous. Let us confirm the relative magnitudes of the terms in $K_{z}$ and $K_{z}^{\prime}$ in Eq. (2.11) (for protons and deuterons). In the next figure, we display the value of $\left|F_{3}\right|$ and also the separate contributions $\left|F_{3 a}\right|$ and $\left|F_{3 b}\right|$, which are obtained by retaining only the terms from $K_{z}$ and $K_{z}^{\prime}$, respectively, in Eq. (2.11). Figure 2 shows the values of $\left|F_{3}\right|,\left|F_{3 a}\right|$, and $\left|F_{3 b}\right|$ as a function of $\nu_{z}$ for protons (left) at $p=$ $2.1 \mathrm{GeV} / c$ and deuterons (right) at $p=1.85 \mathrm{GeV} / c$. It is clear that for protons, $\left|F_{3 a}\right| \gg\left|F_{3 b}\right|$ at all values of $\nu_{z}$, and the total value of $F_{3}$ comes almost entirely from the "magnet body" term $F_{3 a}$. On the other hand, for deuterons $\left|F_{3 a}\right| \ll\left|F_{3 b}\right|$ at all values of $\nu_{z}$, and the total value of $F_{3}$ comes almost entirely from the "fringe field" term $F_{3 b}$. 

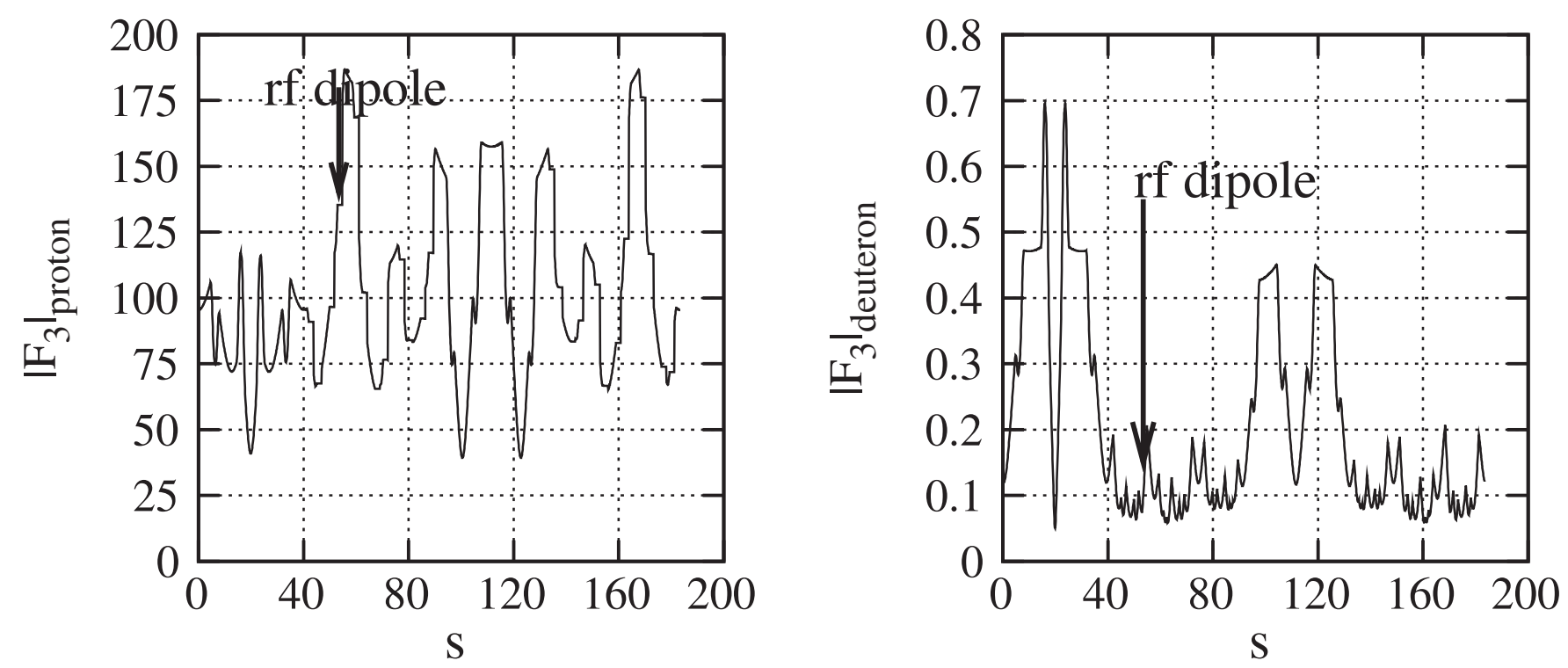

FIG. 1. Graph of $\left|F_{3}\right|$, for protons (left) and deuterons (right), around the circumference of the COSY ring. The proton momentum is $p=2.1 \mathrm{GeV} / c$, and the vertical betatron tune is 3.525 , from [3]. The deuteron momentum is $p=1.85 \mathrm{GeV} / c$ and the vertical betatron tune is 3.60 , from [5]. The location of the rf dipole is indicated.

We now analyze the proton spin-flip data. A graph of the ratio $\mathcal{R}$ as a function of the vertical betatron tune is displayed in Fig. 6 of [3]. The authors showed that the data are well fitted by the functional form

$$
\mathcal{R}_{p}=A_{p}+\frac{B_{p}}{\left|\nu_{r p}-\nu_{z}\right|},
$$

where $\nu_{r p}$ is the resonant tune for the protons. According to [3], the fitted values are

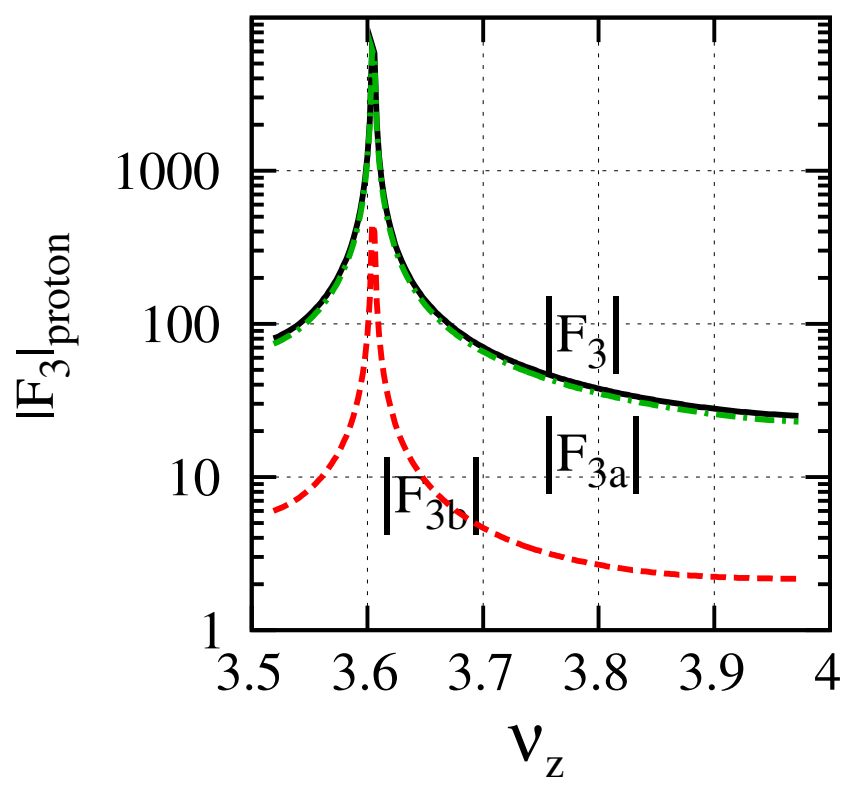

$$
\begin{aligned}
A_{p} & =0.44 \pm 0.46 \\
B_{p} & =0.50 \pm 0.03 \\
\nu_{r p} & =3.6060 \pm 0.0005 .
\end{aligned}
$$

Note that all of the data in [3] were on the left side of the peak, i.e. $\nu_{z}<\nu_{r p}$. Because of the factor of 2 error pointed out by Bai, MacKay, and Roser [6], the values of $A_{p}$ and $B_{p}$ must be multiplied by 2 . Since $p=2.1 \mathrm{GeV} / c$ yields

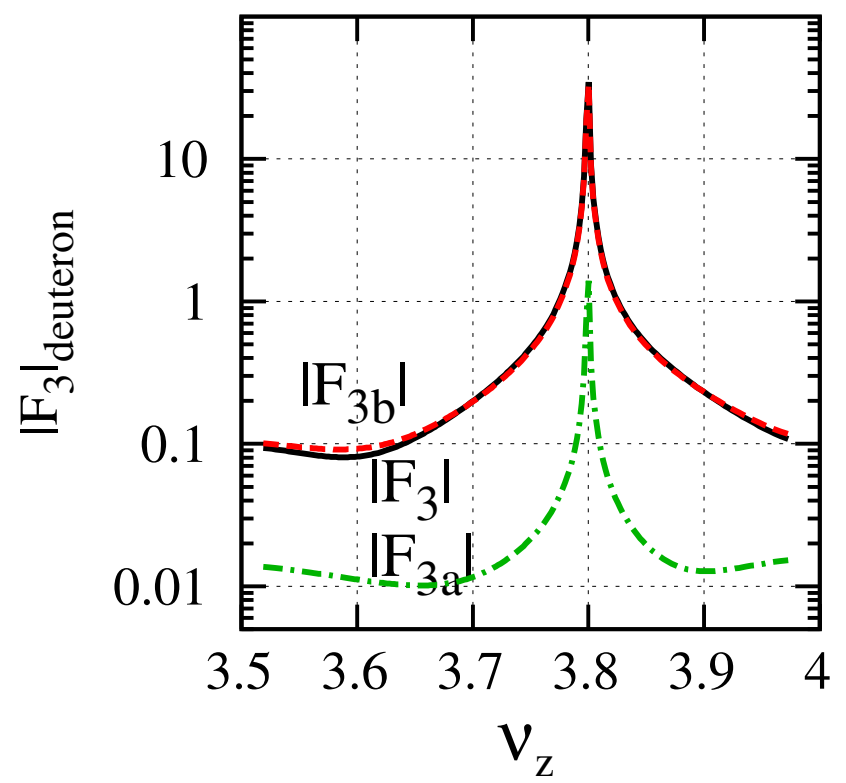

FIG. 2. Graph of $\left|F_{3}\right|$ at the location of the rf dipole as a function of the vertical betatron tune $\nu_{z}$, for polarized protons (left) at $p=2.1 \mathrm{GeV} / c$ and polarized deuterons (right) at $p=1.85 \mathrm{GeV} / c$. The curves for $F_{3 a}$ and $F_{3 b}$ are the individual contributions to $F_{3}$ from the terms in $\left(\nu_{0}^{2}+G\right) K_{z}$ and $(1+G) K_{z}^{\prime}$, respectively, as shown in Eq. (2.11). 
$G \gamma \simeq 4.395$ leading to $8-G \gamma \simeq 3.605$, we reduced the proton momentum slightly to align the resonance peak with the data. Hence, we compare our computed value of $\left|F_{3}\right|$ to the value of

$$
\mathcal{F}_{p}=2(G \gamma+1)\left[A_{p}+\frac{B_{p}}{\left|\nu_{r p}-\nu_{z}\right|}\right],
$$

where $G \gamma \simeq 4.394$. Figure 3 shows a plot of the theoretical value of $\left|F_{3}\right|$ (solid curve) and the above value of $\mathcal{F}_{p}$ (dotdashed curve) against the vertical betatron tune $\nu_{z}$. Our theoretical calculations agree very well with the experimental data.

Next we analyze the deuteron data. A graph of the ratio $\mathcal{R}$ as a function of the vertical betatron tune is displayed in Fig. 4a of [5]. The resonance peak is asymmetric, and the data span both sides of the peak. The authors showed that the data (except for the point at $\nu_{z}=3.6$ ) are well fitted by the functional form

$$
\mathcal{R}_{d}=\left|A_{d}+\frac{B_{d}}{\nu_{r d}-\nu_{z}}\right|
$$

where $\nu_{r d}$ is the resonant tune for the deuterons. According to [5], the fitted values are

$$
\begin{aligned}
A_{d} & =0.06 \pm 0.04, \\
B_{d} & =0.010 \pm 0.002, \\
\nu_{r d} & =3.798 \pm 0.001 .
\end{aligned}
$$

The authors claim in [5] to have addressed the factor of 2 error which was present in the proton data above, hence one should not multiply the values of $A_{d}$ and $B_{d}$ by 2 . Since $G \gamma \simeq-0.20084$ for $p=1.85 \mathrm{GeV} / c$, leading to $4+$ $G \gamma \simeq 3.799$, we increased the momentum slightly to align

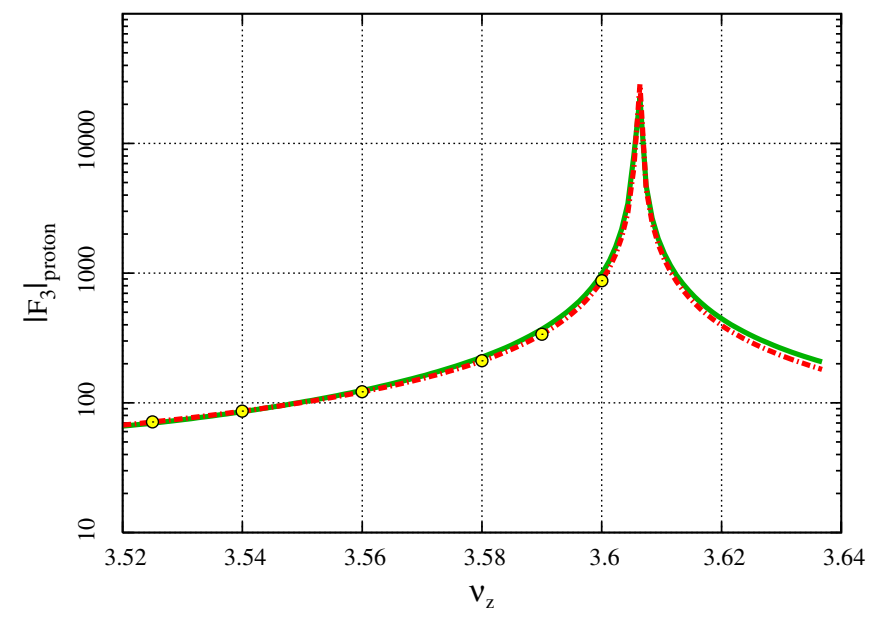

FIG. 3. Graph of $\left|F_{3}\right|$ (for protons, at the location of the rf dipole in COSY) plotted against the vertical betatron tune $\nu_{z}$. The proton momentum is $p=2.1 \mathrm{GeV} / c$. The solid curve is a numerical calculation. The dot-dashed curve is (a factor of 2 times) the experimental fit to the data, published by the authors of [3]. the resonance peak with the data. We thus compare our computed value of $\left|F_{3}\right|$ to the value of

$$
\mathcal{F}_{d}=\left|(G \gamma+1)\left[A_{d}+\frac{B_{d}}{\nu_{r d}-\nu_{z}}\right]\right|,
$$

where $G \gamma \simeq-0.202$. Figure 4 shows a plot of the theoretical value of $\left|F_{3}\right|$ (solid curve) and the above value of $\mathcal{F}_{d}$ (dot-dashed curve) against the vertical betatron tune $\nu_{z}$. Both the ASPIRRIN curve and the experimental curve are asymmetric. The theoretical curve is in general slightly lower than the data, but overall it is a reasonable fit to the data.

Let us discuss the deuteron data in more detail. First, the asymmetry arises from nonresonant background terms. The integral for $F_{3}$ [see Eq. (2.11)] is a sum of many terms, corresponding to different resonance denominators (basically, for $\nu=k \pm \nu_{z}$ for all integers $k$ ). However, not all of the terms are strong. Since for the deuterons $G \gamma \simeq-0.2$, and $\nu_{z}$ goes from 3.6 to 3.85 , the principal resonance denominator is given by $\nu=-4+\nu_{z}$. This denominator approaches zero when $\nu_{z} \simeq 3.8$, and the term in $F_{3}$ corresponding to this denominator is responsible for the peak in Fig. 4. However, the value of $F_{3}$ also contains contributions from terms with resonance denominators at $-3+\nu_{z}$ (far away) and $3-\nu_{z}$ and $4-\nu_{z}$, and others which are even farther away. These denominators do not vanish for a vertical betatron tune scan spanning the interval $3.6<$ $\nu_{z}<3.85$; hence, they contribute to a nonresonant background, leading to the asymmetry of the data in Fig. 4. Next note that, if we compare the data values in Eqs. (3.5) and (3.8), we see that the resonance strength parameter for deuterons $B_{d} \simeq 0.01$ is 50 times smaller than that for protons $B_{p} \simeq 0.50$ (or a factor of 100 if we compare to $2 B_{p} \simeq 1.0$ ). Hence, the deuteron spin-flip resonance is

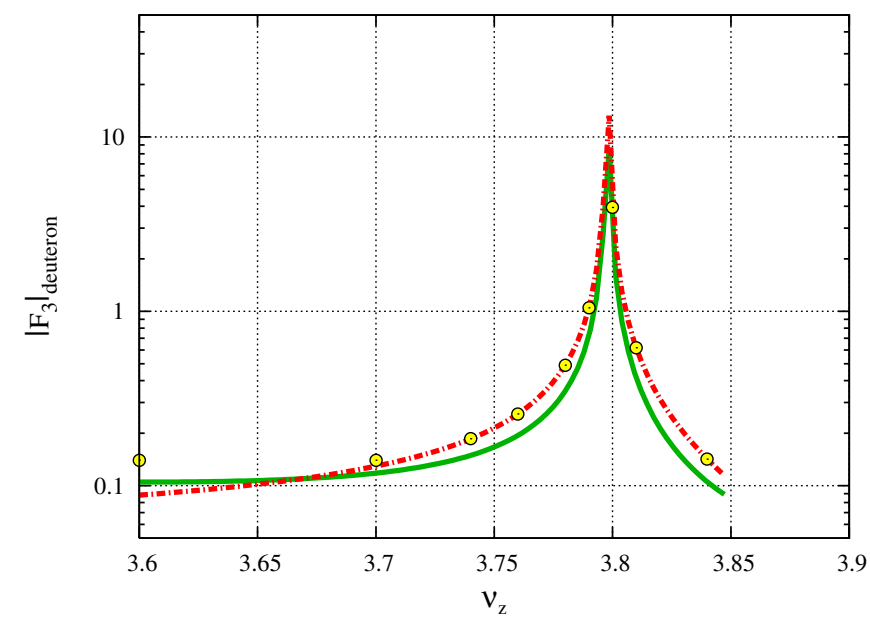

FIG. 4. Graph of $\left|F_{3}\right|$ (for deuterons, at the location of the rf dipole in COSY) plotted against the vertical betatron tune $\nu_{z}$. The deuteron momentum is $p=1.85 \mathrm{GeV} / c$. The solid curve is a numerical calculation. The dot-dashed curve is the experimental fit to the data published by the authors of [5]. 
much weaker, and the effects of nonresonant background terms in the data are more significant. For the proton data, the functional fit in Eq. (3.4) is symmetric by construction, and in any case the resonant term is so strong (note that $\left|F_{3}\right| \geq 100$ in most of Fig. 3) that the contribution from the nonresonant term $A_{p}$ is negligible. In general, it is difficult to quantify the nonresonant background terms in detail. Note that the values of $A_{p}$ and $A_{d}$ both have large uncertainties. We do not know all of the details of the experimental procedure, and so we cannot pursue the matter further.

\section{CONCLUSION}

We calculated the spin-flip resonance strengths, for a radial field rf dipole spin flipper, for stored polarized proton and deuteron beams at COSY. The key point was to include both the direct spin coupling and the indirect spin coupling via the vertical betatron oscillations induced by the rf dipole. The most obvious manifestation of the latter contribution is the appearance of a resonance peak when the resonance strength is plotted as a function of the vertical betatron tune. We used the program ASPIRRIN to perform our numerical calculations. We plotted graphs of the (scaled) resonance strength as a function of the vertical betatron tune, for both polarized protons at $p=$ $2.1 \mathrm{GeV} / c$ and polarized deuterons at $p=1.85 \mathrm{GeV} / c$. We obtained excellent agreement for the polarized proton data, when a factor of 2 error in the data was corrected. The deuteron spin-flip data exhibits considerable asymmetry due to nonresonant background terms. The ASPIRRIN calculation reproduced the overall asymmetry of the deuteron data, and also gave a good fit to the data. We do not know enough details of the experiment to quantify all of the background nonresonant terms which contribute to the asymmetry. However, we have enough evidence to state that there is no "unexpected discrepancy" between theory and measurement for the spin-flip data (for both protons and deuterons).

\section{ACKNOWLEDGMENTS}

We thank A. M. Kondratenko for informing us of his joint work with M. A. Kondratenko and Yu. N. Filatov. We also thank V. Ptitsyn for valuable comments on the spin response formalism and also the ASPIRRIN program, and A. V. Otboev for helpful pertinent insights.

[1] V.S. Morozov et al., Phys. Rev. ST Accel. Beams 7, 024002 (2004).

[2] M. A. Leonova et al., Phys. Rev. Lett. 93, 224801 (2004).

[3] M. A. Leonova et al., Phys. Rev. ST Accel. Beams 9, 051001 (2006).

[4] V.S. Morozov et al., Phys. Rev. ST Accel. Beams 8, 061001 (2005).

[5] A.D. Krisch et al., Phys. Rev. ST Accel. Beams 10, 071001 (2007).

[6] M. Bai, W. W. MacKay, and T. Roser, Phys. Rev. ST Accel. Beams 8, 099001 (2005).

[7] A. M. Kondratenko, Report No. 82-28, 1982.

[8] V. Ptitsyn, Ph.D. thesis, Budker Institute of Nuclear Physics, Novosibirsk, 1997 (in Russian).

[9] E. A. Perevedentsev, Yu. M. Shatunov, and V. Ptitsin, Proceedings of SPIN02, AIP Conf. Proc. No. 675 (American Institute of Physics, New York, 2003), p. 761.

[10] A. M. Kondratenko, M. A. Kondratenko, and Yu. N. Filatov, JINR Report No. P9-2008-16 [Pis'ma Fiz. Elem. Chastits At. Yadra (Phys. Part. Nucl. Lett.) (in Russian)].

[11] L. H. Thomas, Philos. Mag. 3, 1 (1927).

[12] V. Bargmann, L. Michel, and V.L. Telegdi, Phys. Rev. Lett. 2, 435 (1959).

[13] S. R. Mane, Yu. M. Shatunov, and K. Yokoya, Rep. Prog. Phys. 68, 1997 (2005).

[14] M. Froissart and R. Stora, Nucl. Instrum. Methods Phys. Res., Sect. A 7, 297 (1960) (in French).

[15] E.D. Courant and R.D. Ruth, Brookhaven National Laboratory Technical Report No. BNL 51270, 1980.

[16] Ya. S. Derbenev, A. M. Kondratenko, and A. N. Skrinsky, Part. Accel. 9, 247 (1979). 